\title{
Effects of Substrates and Shapes of Cavities on the Gain and Directivity of a Dipole Antenna
}

\author{
Aaron Don M. Africa, Samuel Alexander Pasia, Jereme Adriane Sy \\ Department of Electronics and Communications Engineering \\ De La Salle University, Manila \\ 2401 Taft Ave., Malate, Manila 1004, \\ Philippines, aaron.africa@dlsu.edu.ph
}

\begin{abstract}
Antennas are useful devices as they are used to enable long distances communications. Up until today, there are still researches about designing an to further progress and innovate the said device. There are many ways in improving the antenna such as varying the size and many more. In this research, the students will demonstrate how the substrate and shape of an antenna cavity influence the performance of a dipole antenna. The substrate is an important part of designing antennas as there is no ideal substrate, and different substrates have different applications. In addition to that, the substrate affects the electrical performance of the antenna and its amplifier circuit. For the antenna substrates, the students will use 9 different substrates. For the shapes, the students will use rectangular and circular shapes. Therefore, in the end, the students will test and gather results to determine which of the substrates is the best among them, and which shape of the antenna cavity is better than the other.
\end{abstract}

Key words: antenna, antenna design, antenna cavity, substrates, dipole antenna.

\section{INTRODUCTION}

Antennas are electronic devices that are mainly found in communications, primarily in telecommunications. The word telecommunications came from the Greek word "tele" which means "at a distance", thus it means" distant communications". The antenna has a purpose of radiating, and this radiating will cause electrical signals to reach far distances from the antenna. For its definition, the antenna is a part of a communication system intended to radiate or receive EM waves [1].

An antenna must be optimized and efficient to fully utilize it. There are different performance parameters to check the efficiency of an antenna, such as radiation pattern, directivity, gain, polarization, and impedance. The radiation pattern is the radiation around the antenna, which can be directive, omnidirectional, or shaped. Antenna directivity is the power density ratio of the antenna, which means that a higher directivity is a higher concentration of bean radiation. The antenna gain is the directivity without losses, which determines how good the antenna power in a direction. Polarization is the polarized field by the antenna, which can be linear, elliptical, or circular. Impedance is the input impedance located at the terminals of the antenna. There are also physical parameters that certainly affect how an antenna operates like its size, dimensions, weight, antenna cavity, environmental aspects, nearby conductors [2].

For this research, the students aim to improve the quality of a dipole antenna by introducing different effects of substrates of antenna cavities. With this, the students will simulate different substrates and check whether it will enhance or not the efficiency of a dipole antenna. In addition to that, the students will test different shapes of antenna substrates, which will surely affect how it functions. Lastly, the students will compare the simulated results and determine which type of substrate and shape of antenna has the best efficiency among them.

\section{BACKGROUND OF THE STUDY}

Currently, there are already different works of literature that aim to improve the efficiency of different antennas. Different authors keep on proposing different designs to contribute to the improvement of antennas. They aimed to adjust the different parameters of the antenna to see if there is an effect on performance and efficiency. An example of this is the study Asci and Yegin, where they analyzed the grooves of the antenna [3]. They analyzed that the grooves are often rectangular or triangular because of the limitation of manufacturing. With this, they wanted to determine if the shapes of these grooves affect the performance. They conducted their study by using a waveguide slot fed, dual cavity aperture antenna. They then applied different groove shapes and tested them at the $\mathrm{Ku}$ band. Their antenna achieved a $13.25 \mathrm{dBi}$ peak gain in an operating frequency at $14 \mathrm{GHz}$. Their study successfully showed how different shapes of grooves can affect performance. Another example is the study of Bhattacharya and Maity where they compared three different shapes of microstrip antennas. These shapes 
Aaron Don M. Africa et al., International Journal of Emerging Trends in Engineering Research, 8(9), September 2020, 5392 - 5399

are rectangular, circular, and annular ring shapes. They were able to analyze them by using the cavity mode technique. They set a fixed value for the area of the different shapes of microstrips. They focused only on the geometric properties of the antenna and made all parameters constant [4]. These are only a few of many studies that show how varying the physical properties can still produce an effect on its efficiency. It shows how these antennas can be further improved with different modifications. With this, this study wants to determine the effects of varying the substrate of a cavity that is applied to an antenna. They want to determine if changing the substrates of the cavity can yield a significant change in its efficiency.

\section{STATEMENT OF THE PROBLEM}

Antennas have been around since the 19th century, and even today, these electronic devices are still being researched to further improve their capabilities and potentials. The antennas are known to have problems such as poor reception, low-quality video, and packet loss [5]. Furthermore, some researches enhance the abilities of these electronic devices by simply changing the dimensions which will affect how the antennas work [6]. Additional parameters that can be changed that will affect how an antenna works are the dielectric constant, the substrate, and the antenna cavity. Note that in this research, the students will assume that different limiting factors like physical objects and nearby electric conductors will not be affecting or interfering with the signals it will be receiving.

With the following statements in mind, the students want to research more on improving and innovating the antennas by finding how different types of substrates affect the antenna cavity. This type can be useful as it only has a compact size and suitable for different applications.

\section{SIGNIFICANCE OF THE STUDIES}

The findings of this study will contribute to having better antenna designs. Commercially used antennas can have better performance. It can contribute to a faster approach when it comes to antenna designing. Antenna companies could have a supply of specific substrates that can be used in their antennas. This can help them save time and money as to not use other substrates that would affect the antenna's performance. This study could also be beneficial to antenna designers/hobbyists as it can direct them to a specific way of designing antennas. They would not have a difficult time designing since they can narrow down their choices and would consider fewer inputs for their antenna. It provides them with a simpler method in designing because choosing substrates can never be a problem. Lastly, the results of this study could also be beneficial to college students who are taking the course in engineering. These students could further analyze the properties of the different substrates that cause an effect on the performance of an antenna. They could study the unique characteristics of these substrates and can devise a way on how to alter them. They could also create different studies where this research lacks.

\section{DESCRIPTION OF THE SYSTEM}

For the antenna, most of the parameter is held constant. The operating frequency will be set to $500 \mathrm{MHz}$ which will be the basis of their base model. The dimensions of the antenna as well as the cavity will be constant which is based on the model obtained from MATLAB. Lastly, the substrates to be analyzed are air, FR4, Teflon, foam, fused quartz, Plexiglas, E glass, and polystyrene.

\section{METHODOLOGY}

For this study, the researchers used MATLAB to test the effects of different substrates. They used the antenna designer app to first create a base model of their cavity-backed antenna. They then recorded the measurements of the cavity and coded it in MATLAB as seen in Figure 1. They used the command "cavity()" to set the length, width, height, spacing, and tilt of the cavity. The dipole antenna used for this cavity is the default antenna provided by MATLAB since altering the antenna while changing the substrate produces an error. With this, the substrates were varied by changing a single line of the code while taking note of the changes. This procedure was also repeated for a circular cavity. After getting the results, they analyzed the changes between their gain, directivity.

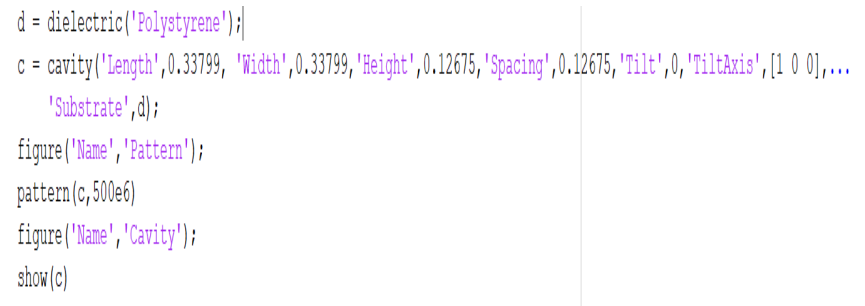

Figure 1: Code used for the Paper

\section{REVIEW OF RELATED LITERATURE}

The study of Mendez et.al designed an antenna with the use of a Groove waveguide technique that can be used with planar technology. Their antenna is made of hard-aluminum and the design of the prototype antenna contains a slotted cavity fed by a microstrip line. Their research made two designs of these antennas. The first one contained three metal walls while the second contained two metal walls. With their new design, they found out that it can be used for hyperthermia treatment [7]. Their study focused on creating a prototype with a groove waveguide technique that produced an acceptable outcome. It consisted of a cavity which helped with the design. For this research, the researchers will be examining the effects of the cavity when attached to an antenna. They will not be doing this to improve equipment from a certain field. 
As per Al-Fadhani et.al they designed a cavity-backed antenna to be applied for satellite communications which have 31-36 GHz Ka-band. The antenna designed is a new type of wideband waveguide (SIW). It is made on RogerRT5880 that has a 2,2-dielectric constant, $1.27 \mathrm{~mm}$ thickness, and a 0.0009 loss tangent. The software used for their simulation was the Microwave studio. With this software, they were able to determine that the antenna operates within 31 to $36 \mathrm{GHz}$ bands. It also achieved an efficiency of $80 \%$ and a gain of 8.87 $\mathrm{dBi}$. This means that the antenna will work properly on the desired application [8]. Since the study utilized a cavity for their antenna, it showed that there are changes with regards to functions of the antenna. Al-Fadhani's team did not indicate how the cavity helped with their study. With this, this research only wants to determine how cavities affect the performance of an antenna.

For dos Santos Silveira, Antreich, and do Nascimento proposed an antenna design that is based on a resonant cavity model. This antenna is a frequency reconfigurable SIW microstrip antenna. They were able to create the design by first gathering different rectangular and triangular cavities. These cavities have walls that are electrically and magnetically perfect. Aside from this, they also created a list for these cavities that can be frequency reconfigurable when combined with other geometries. They did their experimental procedures in an anechoic chamber which was also tested with a software-defined radio GNSS receiver [9]. In their study, they were able to gather different antenna cavities which became their basis for the design of the antenna. They were also able to test it. However, for this research, the researchers used MATLAB to test rectangular and circular cavities of their antennas. These cavities are chosen since they are the only ones available in MATLAB. They only changed the dielectric of these cavities.

Niu and Cao's study also made use of the SIW cavity. They designed a bandwidth-enhanced four-antenna MIMO system that is based on the said cavity. They did this batching two rectangular on top of the cavity and developed four identical quarter-mode-sub-cavities that are connected to a coaxial port to form the antenna. With this, they enhanced the bandwidth by splitting the sub cavity into three parts. These produce three modes for resonance. The final output shows that the bandwidth was changed from 80 to $230 \mathrm{MHz}$ and has better isolation [10]. This study also utilized a cavity in their design. This proved that cavities could improve certain parameters of the antenna.

The study of Fakhte and Aryanian proposed an antenna design that uses that conventional patch alongside. It also includes an artificial magnetic conductor (AMC) ground plane and a frequency selective surface (FSS). Their design made use of circularly polarized waves by using the FSS. To reduce the size of the antenna cavity the AMC ground plane had its phase set to 0 . With this, the antenna shows that it has a $3 \mathrm{~dB} A R$ in the $20 \%$ bandwidth of the antenna while its gain is at $16 \mathrm{dBi}$ [11]. Their study has developed a way to make the cavity more compact by adding different components. For this study, the researchers will not be adding improvements to the cavity.
It will only be a study to analyze the effects of different dielectrics.

As per Xu et.al they have developed a circularly polarized slot antenna with a dual-mode elliptic cavity. The cavity is again a substrate integrated waveguide cavity or SIW. They were able to design by first creating a base model of different distributions of dual-mode cavities. They then divided the antennas into three categories. The first has three unbalanced slots etched into the cavity, the second and third one have both symmetric arc-shaped slots etched into the cavity. To be able to create circularly polarized waves, they activated the 2 quasi modes of the cavity at the same time and combined them with a 5-6 GHz frequency range. With this, their antennas have good aesthetic quality, low cost, good gain, and a simple structure which could benefit in indoor communication [12]. They were able to utilize a cavity in their design. However, since they aim to create circularly polarized waves added procedures are necessary. For this research, the type of waves will not be covered. The researchers will only be focusing on the effect of the cavity on the antenna as a whole.

Banerje and Parui created a SIW based antenna with a circular shaped cavity. This antenna is designed to operate at WLAN operations. This antenna has a rectangular slot placed on the ground plane for radiation. They modified the antenna having an operating frequency of $5.33 \mathrm{GHz}$ and $4.9 \mathrm{dBi}$ gain by placing different dielectric resonators on the slot. Cylindrical shaped and rectangular dielectric resonators provided a 700 $\mathrm{MHz}$ bandwidth enhancement and a hemispherical shape produced a $660 \mathrm{MHz}$ enhancement [13]. Although their research used dielectric resonators, these dielectrics were not applied to the cavities. They did not show what would happen if the cavity had different dielectrics.

Shad and Merphouyan designed a $4 \times 4$-element slot array antenna with an operating frequency of $60 \mathrm{GHz}$. The antenna has high efficiency and high gain and it is designed with a single-layered square quad-ridge cavity structure. Their antenna has a simpler design than conventional antennas because of the lack of power dividers in the feed. A stepped design was also used to enhance the bandwidth and the aperture of the antenna contains a dielectric to decrease the coupling loss of antennas. Their results show that the antenna has a $60 \%$ increase in efficiency and a gain of more than 23.1 sidelobes [14]. Their study showed how dielectrics can be used to reduce the coupling loss. However, they were not able to compare what dielectric could have a better performance.

The study conducted by Raj proposed a SIW based cavity-backed antenna which is also done by previous authors stated in this literature. The difference between Raj's designed antenna is that shorting pins were added to improve gain and cross-polarization. The maximum gain obtained was at $8.6 \mathrm{dBi}$ while its operating frequency is at $10 \mathrm{GHz}$ with a unidirectional directivity [15]. The SIW based cavity-backed antenna has been a common topic in different researches. This is mainly because different authors aim to improve its characteristics. However, for this research, the researchers will not be utilizing the SIW based cavity antennas. 
Dong et al proposed a dipole type millimeter-wave antenna that includes directional radiation characteristics. To increase its bandwidth, a dipole radiation patch and a rectangular parasitic patch was used. However, this was insufficient since they aim to have a directional radiation characteristic. They then achieved this by placing the radiating patch structure about a conducting cavity-backed ground structure. By doing this, they were also able to increase the bandwidth. Their results show that the antenna can have a $10 \mathrm{~dB}$ bandwidth of $51.3 \%$ and is stable. Because of the directional characteristics over the mm-frequency wave band, they said that they can be used for small size wireless mm-wave antennas [16]. Their research was able to increase the characteristics of their antenna by adding additional components to its cavity.

The study of Kumar et al also aimed to improve the performance of the SIW antenna in terms of bandwidth. However, in their case, the antenna is $\mathrm{U}$ shaped. The design they made is applied for $5 \mathrm{G}$ applications that can cover a 28 $\mathrm{GHz}$ band. They stated that the $\mathrm{U}$ shape creates a loading in the slot that is inductive which would lead to an increase in bandwidth performance. Their measurement of their design revealed that the bandwidth reaches $3.9 \mathrm{GHz}$ with a gain of $7.15 \mathrm{dBi}$ [17]. Their study shows how varying the different shapes of antennas can increase its performance. However, they also did not specify how the cavity that was used can affect the antenna. They did not focus on what significance the cavities have when it comes to antenna designs.

As per Jaiswal et al, they also designed an antenna that is to be used for $5 \mathrm{G}$ applications. The antenna they designed is a wideband dual-polarized, cavity-backed microstrip patch antenna. Their design consists of a square patch and two rectangular patches on the top and bottom layers respectively on the substrate. With this, the results show that the antenna has a good return loss bandwidth and isolation that is better than $15.2 \mathrm{~dB}$ [18]. They stated that placing their substrate on the cavity improved the polarization. The researchers want to determine if changing its dielectric can further improve the polarization.

Qi et al proposed a high gain cavity-backed patch antenna. The antennas are based on low temperature cofired technology. They added a substrate integrated cavity to the patch antenna which improved the gain by $3.3 \mathrm{dBi}$. A rectangular ring was also used to improve the impedance matching. With this, the gain of their antenna has reached 9.8 $\mathrm{dBi}$ [19]. Using a substrate cavity improved the gain of the antenna by $3.3 \mathrm{dBi}$. The researchers of this study want to find out what type of substrate could also have a better increase in gain as well as what substrate is best suited for different applications.

Thorat, Wagh, Chandgude, and Prabat designed a microstrip antenna for wireless applications. Their design is a microstrip patch antenna, with substrate dimensions of $40 \mathrm{~mm} \times 35 \mathrm{~mm} \times$ $1.6 \mathrm{~mm}$, patch dimensions of $16 \mathrm{~mm} \times 15 \mathrm{~mm}$, and feed dimensions of $3 \mathrm{~mm} \times 12 \mathrm{~mm}$. Their wanted design has an operating frequency of $3.1 \mathrm{GHz}$ to $10.6 \mathrm{GHz}$. Their results show that the proposed antenna is valid in the said operating frequency band, and has a good antenna gain of $4 \mathrm{dBi}$. Additionally, the results show that the antenna supports UWB, which means it can support large bandwidths [20]. This research is also designing antennas, similar to what the students want to propose. As such, the research can be used as a reference in designing a good antenna.

Casu, Moraru, and Kovacs proposed a paper about designing and implementing microstrip patch antenna arrays. Their specifications are $4 \times 1$ and $8 \times 1$ microstrip patch antenna, the permittivity of dielectric substrate equal to 4.28 , a tangent loss equal to 0.002 , and height equal to $1.6 \mathrm{~mm}$. The designed antenna is designed to use in WLAN and an operating frequency of $2.4 \mathrm{GHz}$. From their simulation results, it can be seen that the micro patch antenna has a smaller bandwidth than the array. However, the values obtained are similar and identical to each other, proving that the micro patch is more direct than the array [21]. The results of this research can be beneficial to the students as they are also into designing antennas. From their research, it can be inferred that micropatch is more direct.

In research by Gan et al, they designed a microstrip antenna array for a $45 \mathrm{GHz}$ application. For this, the designed antenna is a compact, wideband, circularly polarized. Their measured and simulated results displayed that the designed antenna has a bandwidth impedance of greater than 24.4 and an increase of axial ratio bandwidth. In conclusion, the fabricated $4 \times 4 \mathrm{CP}$ antenna has a good performance, a gain of $17 \mathrm{dBi}$, and an operating frequency of $41 \mathrm{GHz}$ to $49 \mathrm{GHz}$ [22].

Imamaoglu and Karpat developed a new design of micropatch antenna for GPS purposes. Their goal is to design such an antenna for vehicular navigation systems. For gathering results, they first simulated a rectangular patch antenna, then simulated a modified patch antenna with circular cavities of the top left and top right of it. The rectangular patch antenna shows that the resonant frequency of it is $1.498 \mathrm{GHz}$, a $10 \mathrm{~dB}$ bandwidth of $135 \mathrm{MHz}$, and VSWR of 1.337. For the modified patch antenna, they found out that increasing the radius means an increasing resonance frequency and increasing $10 \mathrm{~dB}$ bandwidth [23].

In Korea, researchers Kim, Y.H. Kim, Hur, and Woo developed a microstrip antenna design that automatically recovers its resonant frequency and returns loss if it shifts. The said shifting must be approached by physical matters, for instance, the antenna was moved by a hand. To do this, the key is to enable the antenna that can be electronically controlled and frequency reconfigurable. The researchers used micro-controllers for reading, voltage-controlled antenna for recovery. As a result, their design microstrip antenna can automatically recover the resonant frequency and impedance in a matter of seconds [24]. The designed micropatch antenna by the researchers is revolutionary as it can automatically restore its previous state of optimized working conditions. As it also talks about designing antenna, this research can be useful as the students are also designing antennas. 
Researchers Ryu, Jung, and Woo presented a paper where they designed a microstrip antenna for HF and VHF band. Their proposed design is a miniaturized microstrip antenna, with an operating frequency of $40 \mathrm{MHz}$. For their results, they used a standard microstrip antenna to compare their designed antenna. They found out that their designed antenna has a significantly smaller $-10 \mathrm{~dB}$ bandwidth, doubled max gain, and a definite smaller size as it is miniaturized [25]. The results of this research determined that there will still be a better design of microstrip antennas than the standard ones. As such, the students will use this research as a reference in designing their own antenna.

Indonesian researchers Zulkifli, Muhtadin, Basari, and Rahardjo designed a microstrip patch antenna working for LTE applications. They wanted an antenna capable of covering wider bandwidth, while not having a high price. The researchers' antenna is also a MIMO monopole, making it a great antenna for the said purposes. In their simulation and measurement, they found out that the achieved bandwidth of their design is $880 \mathrm{MHz}$ to $2600 \mathrm{MHz}$. Additionally, the radiation pattern displays that the antenna is omnidirectional and a gain of 2 to $5 \mathrm{~dB}[26]$.

$\mathrm{Li}$, Zhang, Joines, and Liu designed a microstrip antenna along with bandwidth enhancement. The researchers intended to design a miniaturized circularly polarized by shorting the strips and patch, then by inductive and capacitive loading. In determining the antenna size, the final antenna dimensions (in millimeters) are $50 \times 50 \times 6.2$. In addition to a smaller size, the designed antenna also exhibits a larger bandwidth, a larger return loss, and an axial ratio of less than $3 \mathrm{~dB}$ [27]. The results of this research show that the designed antenna is better than the standard CMPA ones in the market as it has an enhanced bandwidth and a smaller size.

Pan and Capolino design an antenna with a flat cavity, to enhance the traditional CMOS On-Chip Antenna's radiation pattern and shield its radiating section. Their designed antenna has an area, in millimeter squared, of $1.2 \times 0.6$. From the simulations, it showed that their design has an antenna gain of $-2 \mathrm{dBi}, 20 \mathrm{GHz}$ gain bandwidth, and has $5 \mathrm{GHz}$ bandwidth at $140 \mathrm{GHz}$. Despite having a flat cavity, the designed antenna has a greater efficiency compared. Lastly, due to the cavity, the antenna is not affected by the surrounding conductors [28]. The research by Pan and Capolino further describes what cavities can do to an antenna. They found out that cavities significantly reduce the effective presence of the nearby conductors, and it helps in the efficiency of the antenna itself. This research will be beneficial to the students.

The paper presented by Ouslimani and Meng is a design of an antenna from the Fabry Perot cavity. Their intended design is an antenna for a millimeter-wave band operating at at $80 \mathrm{GHz}$. Using simulation, the researchers found out that the antenna can operate at frequencies between $81 \mathrm{GHz}$ to $86 \mathrm{GHz}$, with a great impedance. The antenna also has a high directivity between the said frequencies and a peak of $38 \mathrm{dBi}$ [29]. This research also tackles the use of cavities in designing antennas.
This paper proves that cavities further help the antenna to be more direct.

Hashmi, Zeb, and Esselle presented a paper about enhancing the bandwidth of the resonant cavity antenna by simply changing the dielectric and thickness constant. To gather their results, they simulated a unit-cell transmission and reflection models. After simulating, they found out that there is a change of bandwidth which is equal to $3 \mathrm{~dB}$ [30]. The results of this paper showed how parameters affect an antenna. Because of this, the students will refer to this paper as they will test different parameters to see what changes will occur in their designed antenna.

\section{THEORETICAL CONSIDERATIONS}

The antenna is an important component as it enables the transmission and reception of signals, which is fundamental in communication systems. This device can also be used by civilians such as mobile communications, and by the military. The research by the students is about enhancing the antenna capabilities by knowing the effect of different substrates and shapes of antenna cavity, which is similar to research in 2005. In this study, the researchers simulated different metamaterials as the substrate to further enhance the gain of a directive antenna [31]. Additional proof of using antenna cavity in an antenna is the designed antenna called cavity mounted dipole antenna. A structure of this antenna and its corresponding polarization is provided below. This proves that this research is feasible in improving the performance parameters of a dipole antenna.

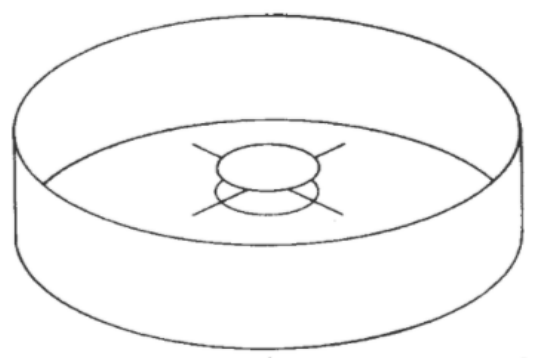

Figure 2: Antenna Cavity

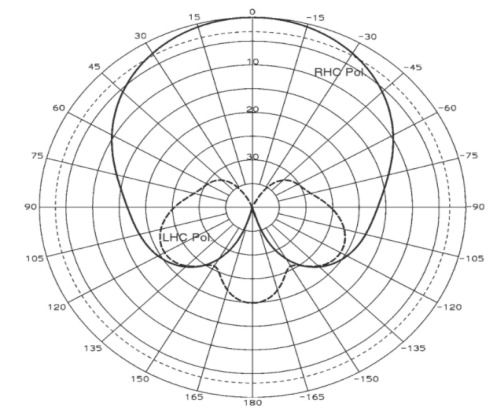

Figure 3: Radiation Pattern

\section{DATA AND RESULTS}

Table 1 shows the maximum gain and minimum gain for the rectangular cavity and the circular cavity. For the rectangular cavity, it can be seen that the substrate that has the highest 
maximum gain obtained is Air followed by Foam, Fused Quartz, Teflon, Polystyrene, Plexiglas, FR4, and E glass.

Table 1: Gains of the Antenna

\begin{tabular}{|c|c|c|c|c|}
\hline & \multicolumn{2}{|c|}{ Rectangular Cavity } & \multicolumn{2}{c|}{ Circular Cavity } \\
\hline Substrate & $\begin{array}{c}\text { Maximum } \\
\text { Gain }\end{array}$ & $\begin{array}{c}\text { Minimum } \\
\text { Gain }\end{array}$ & $\begin{array}{c}\text { Maximum } \\
\text { Gain }\end{array}$ & $\begin{array}{c}\text { Minimum } \\
\text { Gain }\end{array}$ \\
\hline Air & $7.33 \mathrm{dBi}$ & $-27.5 \mathrm{dBi}$ & $7.61 \mathrm{dBi}$ & $-54.4 \mathrm{dBi}$ \\
\hline FR4 & $6.11 \mathrm{dBi}$ & $-14.3 \mathrm{dBi}$ & $3.72 \mathrm{dBi}$ & $-10.3 \mathrm{dBi}$ \\
\hline Teflon & $6.46 \mathrm{dBi}$ & $-20 \mathrm{dBi}$ & $6.89 \mathrm{dBi}$ & $-15.5 \mathrm{dBi}$ \\
\hline Foam & $7.22 \mathrm{dBi}$ & $-34 \mathrm{dBi}$ & $7.59 \mathrm{dBi}$ & $-42.4 \mathrm{dBi}$ \\
\hline Fused Quartz & $6.5 \mathrm{dBi}$ & $-24.9 \mathrm{dBi}$ & $7.46 \mathrm{dBi}$ & $-15.4 \mathrm{dBi}$ \\
\hline Plexiglas & $6.31 \mathrm{dBi}$ & $-20.8 \mathrm{dBi}$ & $6.78 \mathrm{dBi}$ & $-15.1 \mathrm{dBi}$ \\
\hline E-glass & $4.18 \mathrm{dBi}$ & $-29.5 \mathrm{dBi}$ & $5.09 \mathrm{dBi}$ & $-11.7 \mathrm{dBi}$ \\
\hline Polysterene & $6.32 \mathrm{dBi}$ & $-20.8 \mathrm{dBi}$ & $6.79 \mathrm{dBi}$ & $-15.1 \mathrm{dBi}$ \\
\hline
\end{tabular}

It is also important to take note that Air and Foam have one of the smallest minimum gains which means that even though they have a high gain, they are very much susceptible to noise since it has a large back lobe. Plexiglass and Polystyrene on the other hand have almost the same maximum and minimum gains. This means that either one can be used as a substitute if the other choice is unavailable. FR4, Teflon, and Fused Quartz have maximum and minimum gains that are lower than air and foam. What is important here is that the minimum gains are significantly reduced which means that it is less prone to noise. It is more directive. Finally, the E glass has the smallest maximum gain and largest minimum gain which suggests that it is not the best substrate to use. It cannot receive signals properly as well as it is prone to noise. On the other hand, for the circular cavity, the arrangement of the highest to lowest maximum gain is similar to the rectangular, the difference is only found on the last two, where the E-glass has more gain than the FR4. For the arrangement of the highest to lowest minimum gain, the table shows that it starts from FR4, then E-glass, Plexiglas, Plexiglas, Polystyrene, FUsed Quartz, Teflon, Foam, and Air. From the different shapes, it can be seen that the results are almost alike to each other, with the circular-shaped cavity have a greater maximum gain and lesser minimum gain. Therefore, the effect of cavities in the antenna is that it increases both its maximum gain and minimum. The results also show that have a circular-shaped cavity will improve the antennas' maximum gain while also increasing the minimum gain, making the rectangular-shaped cavity a better edge as it also increases the maximum gain while reflecting a small increase of minimum gain.

In determining the best substrate, it is important that the designer knows his/her requirements for a rectangular cavity backed antenna. If the antenna only requires a high gain then air is a suitable choice. On the other hand, if the antenna requires an average performance then FR4 is the best choice since it has a higher minimum gain.

\section{CONCLUSION}

This paper has the aim of improving the efficiency of an antenna by simulating the different effects of various substrates on the performance of the dipole antenna. The intended design of the dipole antenna is a regular dipole antenna, with a cavity that surrounds the antenna. With this, the students tested different types of substrates available in MATLAB, in which they only selected 9 substrates, namely: Air, FR4, Teflon, Foam, Fused Quartz, Plexiglas, E-glass, and Polystyrene. The students tested these substrates, and two types of cavity shapes, which are rectangular-shaped cavity and circular-shaped cavity. After simulating and gathering the data, the students came up with the results and thus analyzed the data gathered. For comparison, the students checked the gain and directivity between each substrate and each shape, therefore there was a total output of 18 results in this paper.

The gathered results showed the maximum gains and minimum gains of the substrates, with the shapes of cavities included. From there, it was seen that the highest maximum gain for both the rectangular-shaped cavity and the circular-shaped cavity is the Air, while the lowest maximum gain for a rectangular cavity is E-glass and for a circular cavity is FR4. Along with these are the minimum gains. The highest minimum gain for each shaped cavity is the FR4, while the lowest minimum gain for the rectangular cavity is foam and for the circular cavity is air.

It is important to know that these gains are the representation of the lobes in a 2D dipole antenna, where the maximum gain is the representation of the main lobe while the minimum gain is the representation of the back lobe. If the designed antenna has a great maximum and minimum $\mathrm{dBi}$, it may not be a recommendation to use that as there is so much power at the back lobe, thus making the antenna lose and waste so much power. Hence in designing the antenna, there must be a balance between the gains and good directivity in order to make up a conventional antenna.

\section{RECOMMENDATIONS}

This study has analyzed the effects of Air, FR4, Teflon, Foam, Fused Quartz, Plexiglas, E glass, and Polystyrene substrates on the rectangular and circular cavity of a dipole antenna. With this, the researchers were able to see how different substrates can affect performance. However, the researchers suggest that the study can be improved by examining the effect of these substrates when the antenna used is of a different type. This could help in determining if the said substrates have the same effect on various antennas. It could show what cavity with a specific substrate to use for a specific type of antenna such as loop, monopole, helix, etc that can give the best performance depending on the requirements. Further study is also needed when the measurements of the antenna are being altered. Since this study made the dimension of the dipole antenna constant, they were not able to examine if the effects of the substrates are uniform for a different size of a dipole antenna. By doing this, it could further improve this research since the dimensions of the dipole antenna will now be fixed or constant for a specific substrate of a specific cavity. This could lead to faster approaches in designing since choosing a substrate of a cavity can already yield to certain measurements of the dipole antenna. Lastly, another area where this research can improve is by analyzing the properties of the substrates. By doing this, it could help determine what other substrates that can be used 
as an alternative. It could also lead to more opportunities in design since it is possible to discover different substrates that could lead to better performance. It could also help when the design needs to be cost-efficient. There can be instances wherein the chosen substrate of the designer does not fit the budget, so he/she has to use cheaper alternatives.

\section{REFERENCES}

[1] R. Garg, P. Bhartia, I. Bahl, and A. Ittipihoon, "Microstrip antenna design handbook," Artect House, 2011.

[2] W. L. Stutzman and G. A. Thiele, "Antenna theory and design," Hoboken, NJ: Wiley, 2013.

[3] Y. Asci and K. Yegin, "Additively manufactured trapezoidal grooves for wideband and high gain $\mathrm{Ku}$-band antenna," International Journal of RF and Microwave Computer-Aided Engineering, vol. 30, no. 3, 2020.

[4] J. Bhattacharya and S. Maity, "A comparative study between rectangular, circular and annular ring shaped microstrip antennas," IEEE Calcutta Conference, CALCON 2020 - Proceedings, pp. 382-385, 2020.

[5] A. Africa, R. Manuel, J. Ligayo, F. Asuncion, J. Tiberio and R. Munchua, "Design of a low-power smart antenna system algorithm," International Journal of Emerging Trends in Engineering Research, vol. 8, no. 8, pp. 4443-4448, 2020.

[6] M. Said, M. Misran, Z. Zakaria, M. Zin and L. Muhammad, "Design of a circularly polarized antenna at $2.45 \mathrm{ghz}$ with harmonic suppression for material characterization," International Journal of Emerging Trends in Engineering Research, vol. 8, no. 8, pp. 4420-4425, 2020.

[7] G. Mendez, H. F., Polochè Arango, M. A., RubianoSuazo, T. A., Rojas Martínez, S. H., andF. Gutiérrez Bernal, "Planar cavity-backed antenna prototype by groove waveguide technique," 2021.

[8] N. Al-Fadhali, H. Majid, R. Omar, M. Ismail, M.Rahim,A. Mumin, and B. Esmail, "Wideband millimeter-wave substrate integrated waveguide cavity-backed antenna for satellites communications," Bulletin of Electrical Engineering and Informatics, vol. 9, no. 5, pp. 1933-1940, 2020.

[9] E. dos Santos Silveira, F. Antreich, and D. Nascimento, "Frequency-reconfigurable SIW microstrip antenna," AEU - International Journal of Electronics and Communications, p. 124, 2020.

[10] B. Niu and Y. Cao, "Bandwidth-enhanced four-antenna MIMO system based on SIW cavity," Electronics Letters, vol. 56, no. 13, pp. 643-645, 2020.

[11] R. Fakhte and I. Aryanian, "Compact fabry-perot antenna with wide $3 \mathrm{~dB}$ axial ratio bandwidth based on FSS and AMC structures," IEEE Antennas and Wireless Propagation Letters, vol. 19, no. 8, pp. 1326-1330, 2020.

[12] Y. Xu, Z. Wang, and Y. Dong, "Circularly polarized slot antennas with dual-mode elliptic cavity," IEEE Antennas and Wireless Propagation Letters, vol. 19, no. 4, pp. 715-719, 2020.
[13] S. Banerjee and S. Parui, "Bandwidth improvement of substrate integrated waveguide cavity-backed slot antenna with dielectric resonators," Microsystem Technologies, vol. 26, no. 4, pp. 1359-1368, 2020.

[14] S. Shad and H. Mehrpouyan, "60 GHz waveguide-fed cavity array antenna by multistepped slot aperture," IEEE Antennas and Wireless Propagation Letters, vol. 19, no. 3, pp. 438-442, 2020.

[15] R. Raj, "SIW cavity-backed patch antenna for X-band applications," Indian Journal of Pure and Applied Physics, vol. 58, no. 3, pp. 164-167, 2020.

[16] Y. Dong, Y.Li, C. Sim, Y. Xia, and X. Liu, "A dipole-type millimeter-wave antenna with directional radiation characteristics," International Journal of RF and Microwave Computer-Aided Engineering, vol. 30, no. 3, 2020.

[17] A. Kumar, S. Bhaskar, and A. Singh, "SIW cavity-backed U-shaped slot antenna for 5G applications," Asia-Pacific Microwave Conference Proceedings, APMC, pp. 1485-1487, 2019.

[18] R. Jaiswal,K.Kumari, and K.Srivastava, "Cavity backed co-radiator dual polarized MIMO antenna," Asia-Pacific Microwave Conference Proceedings, APMC, pp. 792-794, 2019.

[19]Z. Qi, X. Li, J. Chu, J. Xiao, and H. Zhu, "High-gain cavity backed patch antenna arrays at $140 \mathrm{GHz}$ based on LTCC technology," International Journal of Microwave and Wireless Technologies, vol. 11, no. 8, pp. 829-834, 2019.

[20] P. Thorat, P. Chandgude, and P. Wagh,"Design of Microstrip UWB Antenna for Wireless Application," International Journal of Engineering and Technical Research, vol. 9, no.7, 2020.

[21] G. Casu, C. Moraru, and A. Kovacs, "Design and implementation of microstrip patch antenna array," 2014 10th International Conference on Communications (COMM), 2014.

[22]Z. Gan, Z.-H. Tu, Z.-M. Xie, Q.-X. Chu, and Y. Yao, "Compact Wideband Circularly Polarized Microstrip Antenna Array for $45 \mathrm{GHz}$ Application," IEEE Transactions on Antennas and Propagation, vol. 66, no. 11, pp. 6388-6392, 2018.

[23] F. Imamoğlu and E. Karpat, “A new design micropatch antenna for GPS applications on vehicle navigation systems," International Journal of Automotive Engineering and Technologies, vol. 8, no. 4, pp. 172-177, 2019.

[24] Y.-R. Kim, Y.-H. Kim, M.-J. Hur, and J.-M. Woo, "Design of UHF Band Microstrip Antenna for Recovering Resonant Frequency and Return Loss Automatically," The Journal of Korean Institute of Electromagnetic Engineering and Science, vol. 24, no. 3, pp. 219-232, 2013.

[25] H.-K. Ryu, G. Jung, and J.-M. Woo, "A small quarter wavelength microstrip antenna for $\mathrm{HF}$ and VHF band applications," 2010 10th Mediterranean Microwave Symposium, 2010.

[26] F. Zulkifli, N. Muhtadin, Basari and E. Rahardjo, "MIMO monopole microstrip antenna for LTE," 2017 
Aaron Don M. Africa et al., International Journal of Emerging Trends in Engineering Research, 8(9), September 2020, 5392 - 5399

International Symposium on Antennas and Propagation (ISAP), Phuket, 2017, pp. 1-2, 2017.

[27] J. Li, A. Zhang, W. T. Joines and Q. H. Liu, "A miniaturized circularly polarized microstrip antenna with bandwidth enhancement," IEEE International Symposium on Antennas and Propagation (APSURSI), Fajardo, 2016, pp. 41-42,2016.

[28] S. Pan and F. Capolino, "Design of a CMOS On-Chip Slot Antenna With Extremely Flat Cavity at $140 \mathrm{GHz}$," in IEEE Antennas and Wireless Propagation Letters, vol. 10, pp. 827-830, 2011.

[29] H. H. Ouslimani and F. Meng, "Design of Large-Band Highly Directive Antenna in the Millimeter Waves Range at 80 GHz," 2019 IEEE International Symposium on Antennas and Propagation and USNC-URSI Radio Science Meeting, Atlanta, GA, USA, 2019, pp. 1097-1098,2019.

[30] R. M. Hashmi, B. A. Zebt and K. P. Esselle, "Effective techniques for extending the directivity-bandwidth of resonant cavity antennas," International Workshop on Antenna Technology: Small Antennas, Novel EM Structures and Materials, and Applications (iWAT), Sydney, NSW, pp. 270-272,2014.

[31] B.-I. Wu, W. Wang, J. Pacheco, X. Chen, T. M. Grzegorczyk, and J. A. Kong, "A Study Of Using Metamaterials As Antenna Substrate To Enhance Gain," Progress In Electromagnetics Research, vol. 51, pp. 295-328, 2005. 